\title{
NONLINEAR PREDICTIVE CONTROL OF A SOLAR PLANT BASED ON REDUCED COMPLEXITY MODELS
}

\author{
J. M. Igreja ${ }^{*}$ J. M. Lemos ${ }^{* *}$ R. N. Silva ${ }^{* * *}$ \\ ${ }^{*}$ INESC-ID/ISEL, Lisbon, Portugal \\ ** INESC-ID/IST, Lisbon, Portugal \\ *** UNL, Monte da Caparica, Portugal
}

\begin{abstract}
This paper is concerned with predictive controller design for the outlet oil temperature in distributed collector solar fields. The design method proposed combines feedback linearization cascade with an outer loop consisting of a predictive controller incorporating constraints. A relevant feature is the use of a reduced complexity model, selected according to a nonlinearity measure. Copyright $\odot 2005$ IFAC
\end{abstract}

Keywords: Process control, Predictive Control, Nonlinear Systems, Nonlinearity measure, Reduced Complexity Models, Solar plants.

\section{INTRODUCTION}

This paper is concerned with predictive controller design in the presence of constraints for the outlet oil temperature in distributed collector solar fields. Studying this problem is important not only because of its intrinsic interest, but also because it forms a paradigm which can be applied to other processes of economic interest, which involve transport of mass and/or energy over a spatial dimension. Although there is a rich literature on solar plant control (see (Silva et al., 2003; Camacho et al., 1997) for detailed reviews), the problem of incorporating constraints received little attention. In (Camacho et al., 1994) and in other earlier papers there quoted, a static series feed-forward block is used to approximately compensate plant nonlinearity. Both (Camacho, 1994; Coito et al., 1997) consider predictive adaptive controllers, but the focus is on adaptive algorithms which can be applied to a wide variety of plants, and thereby constraints are not discussed. In this paper, instead, the dominant dynamics of the plant is explored in order to propose a predictive control law with constraints.

\footnotetext{
1 This work has been done under the project AMBIDISC, contract POSI/SRI/36328/2000.
}

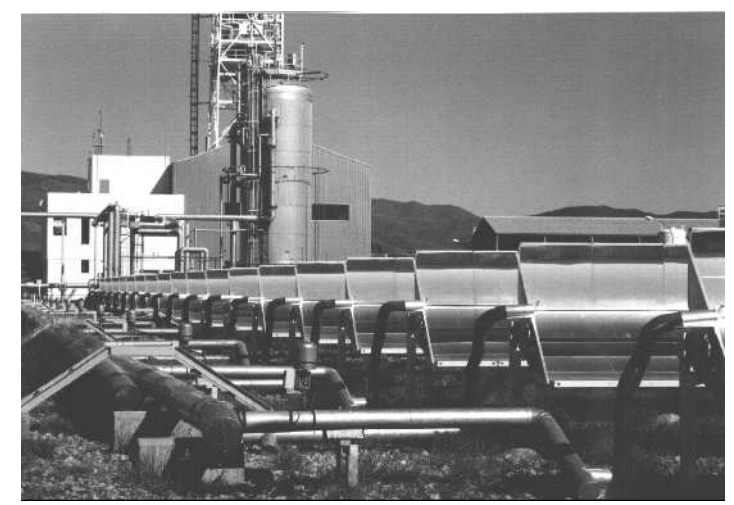

Fig. 1. General view of the ACUREX field.

The control law proposed relies on two main steps: First, a transformation of the manipulated variable is found and shown to yield a reduced complexity linear model. In the second step this model is used to solve a predictive control problem in the presence of constraints. The reduced complexity model is justified here on the basis of a nonlinearity measure as proposed in (Schweickhard et al., 2003; Schweickhard et al., 2003a). This leads to an increased performance. An adaptive version is also presented.

The paper is organized as follows: After this introduction, in which the problem is motivated and the 
main contribution is stated, the reduced plant model is derived in section 2. Section 3 explains how to tackle constraints when using the reduced model and section 4 presents simulation results illustrating the advantage of the proposed approach. Finally, section 4 draws conclusions.

\section{REDUCED ARX FIELD MODEL}

The distributed collector solar field serving as a prototype to this work (fig. 1) is formed by mirror collectors which concentrate direct sun radiation. At their focus lies a pipe containing an oil able to accumulate energy in the form of heat. The main control objective is to control the outlet oil temperature, the manipulated variable being the oil flow. The main disturbance is the solar radiation. Such a plant may be represented by the simplified model (Barão et al., 2002):

$$
\frac{\partial T(z, t)}{\partial t}+u(t) \frac{\partial T(z, t)}{\partial z}=\alpha R(t)
$$

where $T(z, t)$ is the temperature at position $z \in[0, L]$ measured along the pipe at time $t \in[0,+\infty[, u$ is the oil velocity (proportional to flow) and $R$ denotes solar radiation. The parameter $\alpha$ depends on the mirror efficiency and on oil specific heat (which is a function of temperature), being thereby considered as uncertain and $L$ is the pipe length.

Let the pipe be divided in $N$ elements and $h=$ $L / N$. Finite difference approximation of space partial derivatives, yields the following non-linear state-space model:

$$
\dot{x}=\frac{u}{h} A x+\frac{u}{h} B x_{0}+C R(t)
$$

with $A, B$ and $C$ appropriate matrices and the component $k$ of $x$ is $x_{k}(t) \equiv T(k h, t)$. For $k=N$ :

$$
\dot{x}_{N}=-\frac{u}{h}\left(x_{N}-x_{N-1}\right)+\alpha R(t)
$$

where $y=x_{N}=T_{N}$ is the outlet oil temperature that is taken as the model output. Exact input/output feedback linearization (Barão, 2002) is performed by defining the virtual control variable $v$, related to $u$ by

$$
u=\frac{\alpha R-v}{x_{N}-x_{N-1}} h
$$

This transformation puts the system in the ByrnesIsidori normal form (Isidori, 1995) which, since in this case the relative degree is 1 , is an integrator:

$$
\dot{x}_{N}=v
$$

The approximation

$$
\frac{x_{N}-x_{N-1}}{h} \approx \frac{y-y_{0}}{L}
$$

in which $y \equiv x_{N} \equiv T_{N}, y_{0} \equiv x_{0} \equiv T_{\text {in }}$ allows to tackle the technological constraint according to which temperature is only measured at the inlet and the outlet points of the pipe. Furthermore, since

$$
\alpha=\alpha_{0}+\tilde{\alpha}
$$

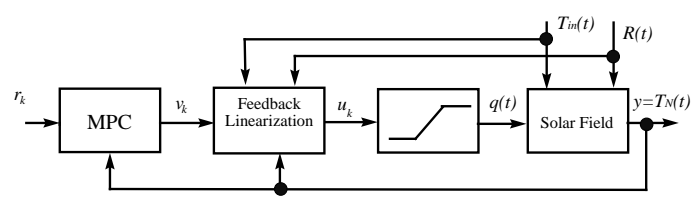

Fig. 2. Controller structure

where $\alpha_{0}$ is the nominal efficiency, yields the approximate feedback linearization law (Barão, 2002):

$$
u=\frac{\alpha_{0} R-v}{y-y_{0}} L
$$

under which (assuming (6)) the field dynamics becomes a pure integrator with a disturbance

$$
\dot{y}=v+\tilde{\alpha} R
$$

The expression (8) corresponds to an exact linearization only for a reduced first order model. When applied to the actual system, its effect is to improve the "linearity" of the resulting compensated system, in the sense of reducing a Nonlinearity Measure (NLM) (Schweickhardt, 2003; Schweickhardt, 2003a). As can be seen in fig. 13 and is discussed in Appendix I, the actual behavior of the system with feedback is not exactly an integrator but can be approximated by an integrator with an extra real pole. Therefore, the compensated system is described by the family of linear systems

$$
\tau \ddot{y}+\dot{y}=K_{p} v+\tilde{\alpha} R(t)
$$

parameterized by $\tau, K_{p}$ and $\tilde{\alpha}$. Remark that, for $\tau=0, K_{p}=1$ and $\tilde{\alpha}=0$ one gets $\dot{y}=v, i$. e. the reduced linearized model with $\alpha=\alpha_{0}$ belongs to the set of models considered. As shown in Appendix I, the inclusion of the extra filter in (10) is made, according to (Schweickhardt, 2003; Schweickhardt, 2003a), by showing that a Nonlinearity Measure is improved for values of $\tau \neq 0$ e $K_{p} \neq 1$.

Fig. 2 shows a block diagram of the proposed controller. Coupling the static compensator defined by (9) with the plant defines a new manipulated variable $v$. Between the new manipulated variable $v$ and the output $y$, it is shown above that the system is described by the linear model (10). For the sake of designing an external loop with a model predictive controller (MPC), (10) is sampled, yielding the ARX model:

$$
y_{k}=a_{1} y_{k-1}+a_{2} y_{k-2}+b_{1} v_{k-1}+d_{1} R_{k-1}+e_{k-1}
$$

The parameters of (11) are related to the parameters of (10) by the expressions

$$
\begin{aligned}
& a_{1}=\frac{2 \tau+d t}{\tau+d t} ; a_{2}=-\frac{\tau}{\tau+d t} \\
& b_{1}=\frac{K_{p} d t^{2}}{\tau+d t} \quad ; d_{1}=\frac{\tilde{\alpha} d t^{2}}{\tau+d t}
\end{aligned}
$$

If an adaptive version is required, these parameters may also be estimated on-line by RLS. 


\section{PREDICTIVE CONTROL WITH CONSTRAINTS}

The predictive controller designed (MPC in fig. 2) minimizes the following quadratic cost

$$
J=\sum_{i=N_{1}}^{N_{2}}\left(r_{k+i}-y_{k+i}\right)^{2}+\rho \sum_{i=0}^{N_{v}-1}\left(\Delta v_{k+i}\right)^{2}
$$

where $r_{k}$ is the reference to track, $y_{k}$ is the output, $\Delta v_{k}$ is the consecutive increment of the input $v$ and $N_{1}, N_{2}, N_{u}\left(N_{2} \geq N_{1}, N_{2} \geq N_{u}\right)$ are integer horizons. The parameter $\rho$ penalizes sudden input excursions. In order to ensure closed loop stability it is possible (Mosca, 1995) to impose $P$ terminal equality constraints in the output:

$$
\begin{aligned}
y_{k+N_{2}+1} & =r_{k+N_{2}+1} \\
& \vdots \\
y_{k+N_{2}+P} & =r_{k+N_{2}+1}
\end{aligned}
$$

In the case where there are additional input and output constraints

$$
\begin{array}{cc}
\underline{u} \leq u(t) \leq \bar{u} & \forall t \\
\Delta u \leq \Delta u(t) \leq \overline{\Delta u} & \forall t \\
\underline{y} \leq y(t) \leq \bar{y} & \forall t
\end{array}
$$

the solution is obtained by solving an equivalent Quadratic Programming (QP) optimization problem that will have a numerical solution if it is feasible. Eventual non-feasible solutions at startup are solved by resorting to the QP solution with no constraints and saturation in $u$.

Since the manipulated variable is now the virtual control $v$ and not $u$, the most significative aspect of the extension is the way in which constraints in $u$ are transformed in constraints in $v$. The constraints in $v$ are time varying and thereby vary along the horizon $N_{v}$, since they depend on future values of input and output. The simplest way to overcome this problem is to obtain the constraints for time $k$ and assume consistent values for the remaining horizon, yielding for the problem at hand:

$$
\begin{array}{ll}
\frac{v_{k 0}}{v_{i}} \leq v_{k} \leq \overline{v_{k 0}} & i=0 \\
\frac{y_{k+i} \leq \overline{v_{i}}}{y_{i}} \leq y_{k+i} \leq \overline{y_{i}} & i=1, \ldots, N_{v}-1 \\
\frac{y_{k+N_{2}+i}=r_{k+N_{2}+1}}{} & i=1, \ldots, P
\end{array}
$$

where

$$
\begin{aligned}
& \underline{v_{k 0}}=\alpha_{0} R-\frac{\left(y-y_{0}\right)}{L} \overline{u_{k}} \\
& \overline{v_{k 0}}=\alpha_{0} R-\frac{\left(y-y_{0}\right)}{L} \underline{u_{k}}
\end{aligned}
$$

Here, $\bar{\omega}$ and $\underline{\omega}$ denote, respectively, the upper and lower allowed bounds of a generic variable $\omega$.

The output constraints are the same since this variable is not transformed by feedback linearization.
Radiation $R$ is seen as a disturbance assumed constant along the prediction horizon $N_{2}$.

\section{RESULTS}

The examples considered hereafter use a rigorous physically based model of the field, developed according to (Camacho, 1997), thereby reproducing with fidelity the nonlinear dynamics of the plant. Inlet oil temperature $T_{i n}(t)$ and radiation $R(t)$ are sequences actually measured in the field. At about $4 \mathrm{~h} 30$ radiation suddenly drops due to a passing cloud, thereby causing a fast disturbance. Three examples are shown for the closed loop.

\subsection{Example 1: Integrator model}

In the first example, the predictive ARX model based on the pure integrator is used, its parameters being kept constant at a priori values. Table 1 shows the controller parameters used, including the horizons in the cost function $N_{1}, N_{2}$ and $N_{v}$, the number of equality constraints $P$, the weight $\rho$, the forgetting factor $\lambda$ for RLS (when used), the value of the input and output constraints, the parameters of the predictive filter $\tau$ and $K_{p}$, the sampling interval $d t$ and the nominal mirror efficiency $\alpha_{0}$.

Fig. 3 shows (from top to bottom) the outlet oil temperature $\left[{ }^{\circ} \mathrm{C}\right]$ and the respective reference signal (above), the corrected solar radiation $\left[W^{-2}\right]$, multiplied by 0.2 , the oil flow $u\left[\mathrm{~m}^{3} \mathrm{~s}^{-1}\right]$, multiplied by $1 \times 10^{5}$ and the virtual control signal, $v,\left[{ }^{\circ} \mathrm{Cs}^{-1}\right]$ multiplied by the factor $1 \times 10^{3}$. Fig. 4 shows the time varying constraint imposed to the virtual signal (dashed lines show the minimum and maximum values allowable to $u$ ), which ensures that the actual flow stands between 2 and $10 \mathrm{ls}^{-1}$. This fact is illustrated in fig. 3 , in which it can be seen that the flow does not violate the lower limit of $2 \mathrm{ls}^{-1}=2 \times 10^{-3} \mathrm{~m}^{3} \mathrm{~s}^{-1}$ when the set-point changes from $225^{\circ} \mathrm{C}$ to $270^{\circ} \mathrm{C}$ just after $5 h$. Fig. 5 shows a detail of the response to setpoint changes, which exhibits an oscillatory character.

\subsection{Example 2: Modified model}

In the second example, table 2 and figures 6,7 and 8 , adaptation is turned on after $1.5 h$, there are no equality constraints and the maximum temperature limit is set to $265^{\circ} \mathrm{C}$. The time constant $\tau$ in (10) is now made different from zero, opposite to the previous example. In fig. 6 a reduction in oscillation is noticeable, thereby suggesting that the extra filter and the adaptation substantially improve controller performance. Remark that the reference, around $5 h$, surpasses the maximum limit imposed to temperature, but the actual temperature respects the constraint (fig. $6)$. 
Table 1. Controller parameters - Example 1.

\begin{tabular}{|l|c|}
\hline Parameter & Value \\
\hline$N_{1}$ & 1 \\
$N_{2}$ & 25 \\
$N_{v}$ & 10 \\
$P$ & 2 \\
$\rho$ & $2 \times 10^{5}$ \\
$\lambda$ & - \\
\hline$\underline{v}\left[{ }^{\circ} \mathrm{Cs}^{-1}\right]$ & -1.0 \\
$\bar{v}\left[{ }^{\circ} \mathrm{Cs}-1\right]$ & 1.0 \\
$y\left[{ }^{\circ} \mathrm{C}\right]$ & 10.0 \\
$\overline{\bar{y}}\left[{ }^{\circ} \mathrm{C}\right]$ & 275.0 \\
\hline$K_{p}[\mathrm{adim}]$. & 1.0 \\
$\tau[s]$ & 0.0 \\
\hline$d t[s]$ & 15.0 \\
$\alpha_{0}\left[{ }^{\circ} \mathrm{Cm}^{2} J^{-1}\right]$ & $4.778 \times 10^{-4}$ \\
\hline
\end{tabular}

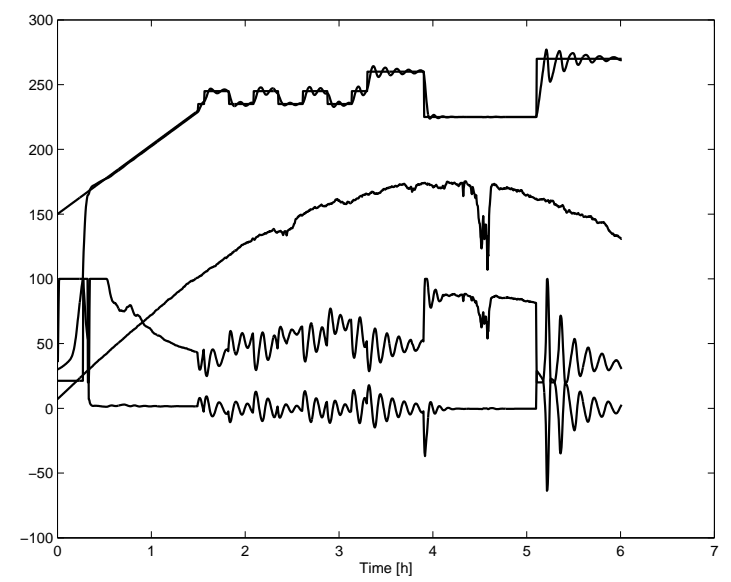

Fig. 3. Example 1 - Process signals.

\subsection{Example 3: Adaptation}

In the last example, table 3 and figures 9, 10, 11 and 12 , the controller is adaptive (the parameters in (11) being estimated by RLS), has equality constraints and the prediction horizon is smaller such as to render it more "aggressive". The controller presents a good performance. Remark in particular the large amplitude transition between $225^{\circ} \mathrm{C}$ and $290^{\circ} \mathrm{C}$ in which the virtual control signal attains the constraint without violating it for a long period of time.

In all simulations there is a very good rejection of disturbances imposed by changes in radiation due to clouds (between $4 h$ and $5 h$.

\section{CONCLUSIONS}

A control algorithm combining feedback linearization, predictive control with constraints and parameter adaptation is applied to temperature control in a distributed collector solar field. The input transform associated to feedback linearization implies a nontrivial constraint management. A distinguishing feature of the work reported consists of using a reduced complexity model justified on the basis of a nonlinearity measure. This lead to a noticeable increase in perfor-

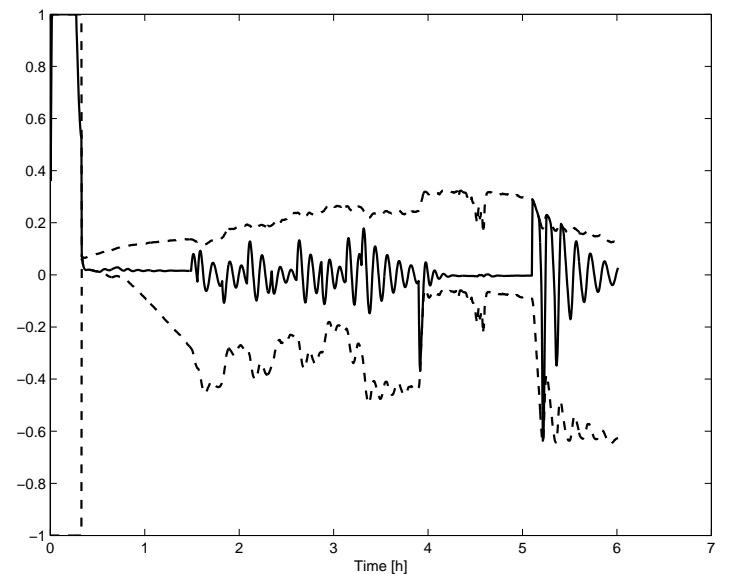

Fig. 4. Example 1 - Time varying constraint in virtual control.

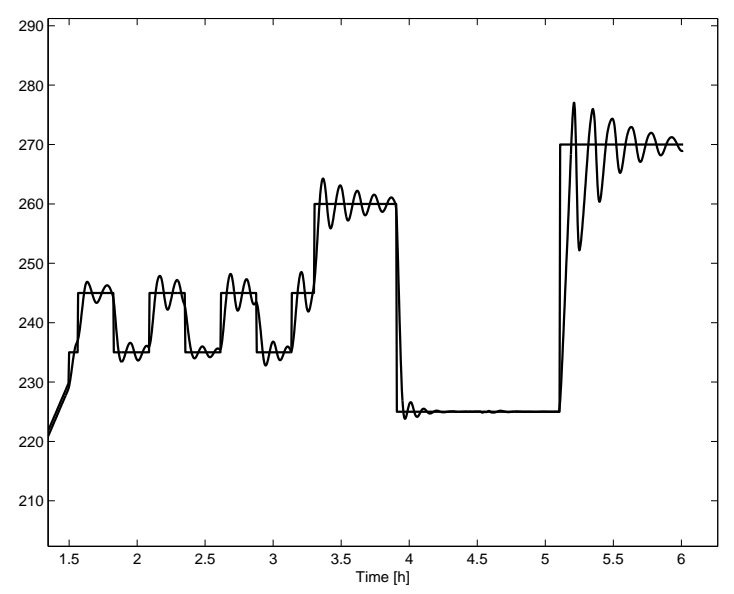

Fig. 5. Example 1 - Detail of temperature response.

Table 2. Example 2 - Controller parameters.

\begin{tabular}{|l|c|}
\hline Parameter & Value \\
\hline$N_{1}$ & 1 \\
$N_{2}$ & 25 \\
$N_{v}$ & 10 \\
$P$ & - \\
$\rho$ & $2 \times 10^{5}$ \\
$\lambda$ & 0.98 \\
\hline$\underline{v}\left[{ }^{\circ} \mathrm{Cs}^{-1}\right]$ & -1.0 \\
$\bar{v}\left[{ }^{\circ} \mathrm{Cs}^{-1}\right]$ & 1.0 \\
$y\left[{ }^{\circ} \mathrm{C}\right]$ & 10.0 \\
$\overline{\bar{y}}\left[{ }^{\circ} \mathrm{C}\right]$ & 265.0 \\
\hline$K_{p}[\mathrm{adim}]$. & 1.0 \\
$\tau[s]$ & 50.0 \\
\hline$d t[s]$ & 15.0 \\
$\alpha_{0}\left[{ }^{\circ} \mathrm{Cm}^{2} J^{-1}\right]$ & $4.778 \times 10^{-4}$ \\
\hline
\end{tabular}

mance (compare figs. 5 and 12), allowing large setpoint jumps $\left(65^{\circ} \mathrm{C}\right)$.

\section{Appendix I - Nonlinearity measure}

This appendix considers the computation of the nonlinearity measure defimned in (Schweickhardt, 2003; Schweickhardt, 2003a) for the reduced model of the linearized solar field. Consider a non-linear BIBO sta- 


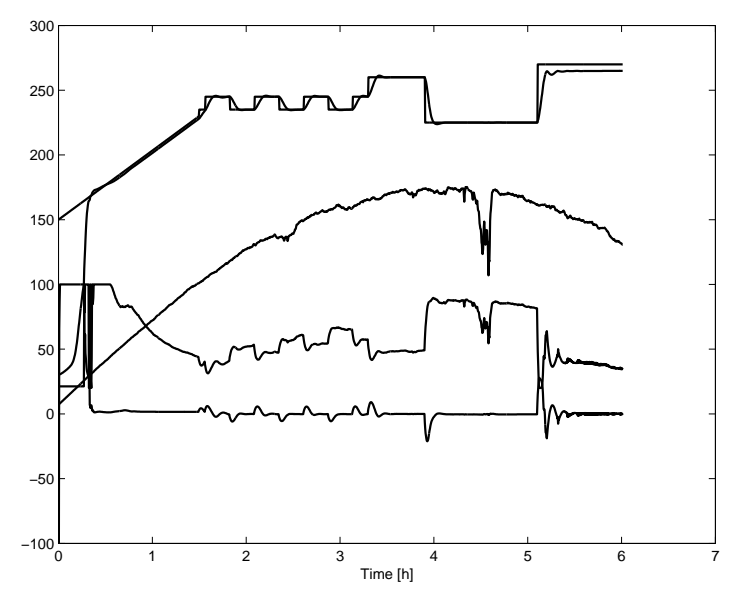

Fig. 6. Example 2 - Process signals.

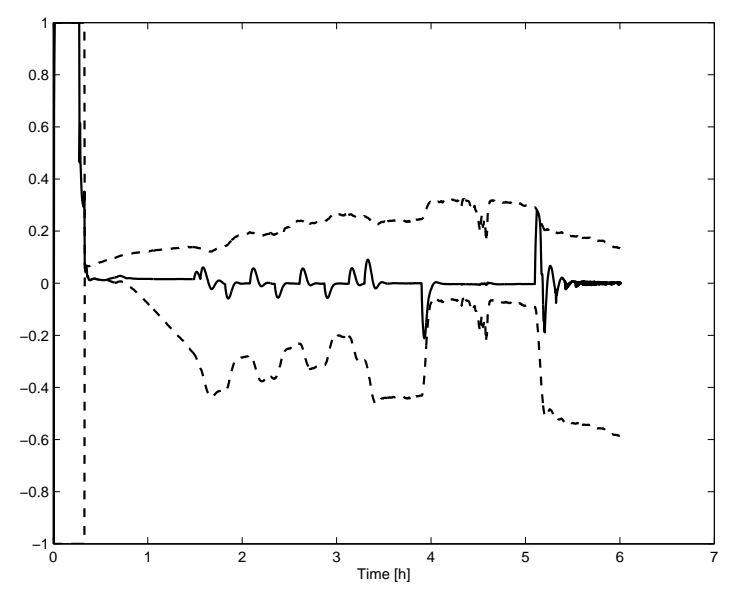

Fig. 7. Example 2 - Time varying constraint in virtual control.

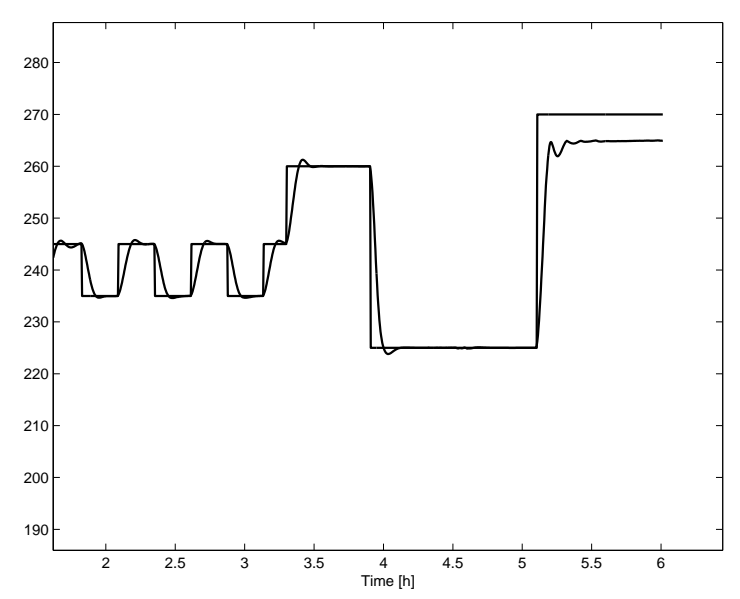

Fig. 8. Example 2 - Detail of temperature response.

ble system described by the operator $y(t)=\mathcal{N}[u(t)]$ which maps its input/ouput behavior. The nonlinearity measure (NLM) $\phi(\mathcal{N}, \mathcal{U})$ of this system is the positive definite scalar given by:

$$
\phi(\mathcal{N}, u)=\inf _{G} \sup _{u} \frac{\|G[u]-\mathcal{N}[u]\|}{\|\mathcal{N}[u]\|}
$$

where $G \in \mathcal{G}$ and $u \in \mathcal{U}, \mathcal{G}$ being the space of linear operators and $\mathcal{U}$ the set of inputs considered.
Table 3. Example 3 - Controller parameters.

\begin{tabular}{|c|c|}
\hline Parameter & Value \\
\hline$N_{1}$ & 1 \\
\hline$N_{2}$ & 15 \\
\hline$N_{v}$ & 10 \\
\hline$P$ & 2 \\
\hline$\rho$ & $5 \times 10^{4}$ \\
\hline$\lambda$ & 0.9999 \\
\hline$\underline{v}\left[{ }^{\circ} \mathrm{Cs}^{-1}\right]$ & -1.0 \\
\hline $\bar{v}\left[{ }^{\circ} \mathrm{Cs}^{-1}\right]$ & 1.0 \\
\hline$\underline{y}\left[{ }^{\circ} \mathrm{C}\right]$ & 10.0 \\
\hline$\overline{\bar{y}}\left[{ }^{\circ} C\right]$ & 295.0 \\
\hline $\begin{array}{l}K_{p}[\text { adim. }] \\
\tau[s]\end{array}$ & $\begin{array}{c}1.0 \\
50.0\end{array}$ \\
\hline $\begin{array}{l}d t[s] \\
\alpha_{0}\left[{ }^{\circ} \mathrm{Cm}^{2} J^{-1}\right]\end{array}$ & $\begin{array}{c}15.0 \\
4.778 \times 10^{-4}\end{array}$ \\
\hline
\end{tabular}

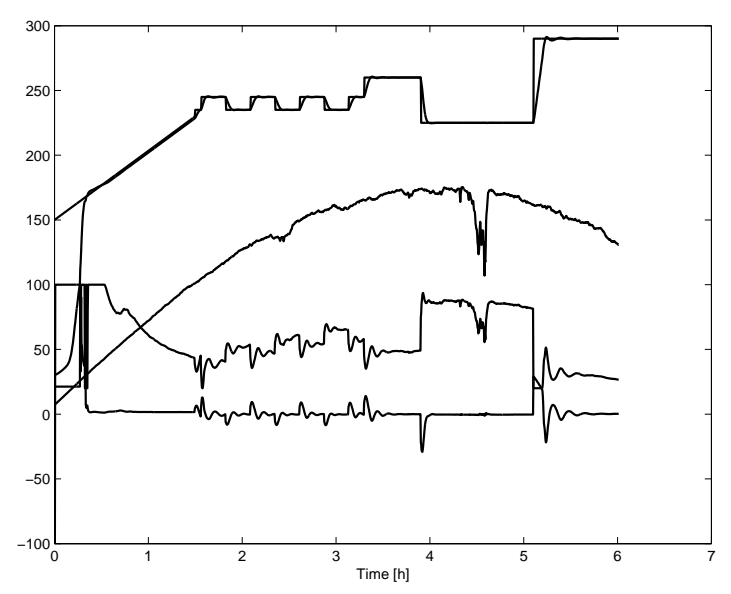

Fig. 9. Example 3 - Process signals.

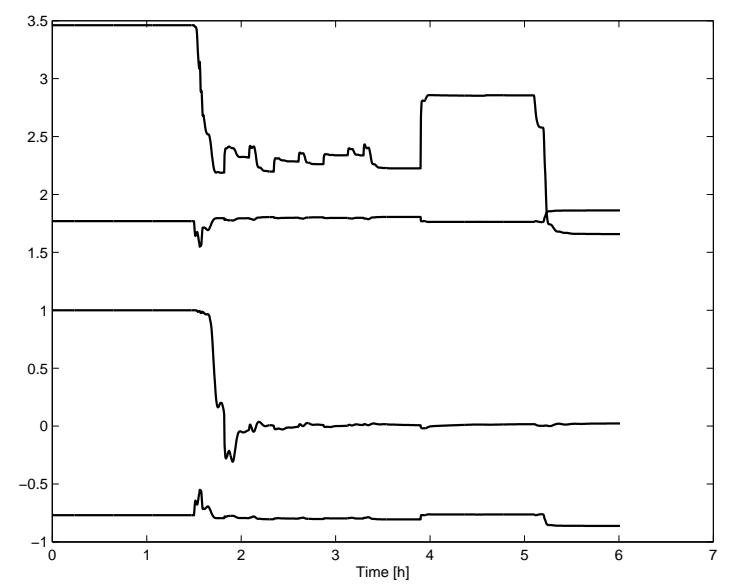

Fig. 10. Example 3 - Adaptive parameters.

The computation of the NLM is in general a difficult, non-linear, min-max optimization problem of infinite dimension. In the case at hand a numerically feasible solution $\phi_{N}$ was obtained restricting $G$ to be given by (10) and the input to be a square wave. The optimization operates therefore only in $K_{p}$ and $\tau$. The nonlinear solution $y_{k}=\mathcal{N}\left[u_{k}\right]$ is obtained by numerical integration of the detailed physical model with feedback, sampled at time $k$. Fig. 13 shows the response of the linearized system, of the pure integrator (which yields a triangular wave) and of the integrator with the 


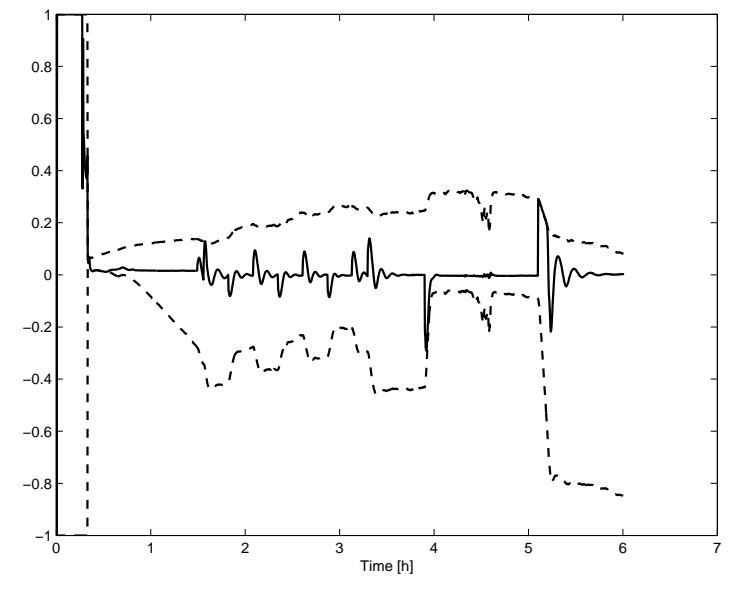

Fig. 11. Example 3 - Time varying constraint in virtual control.

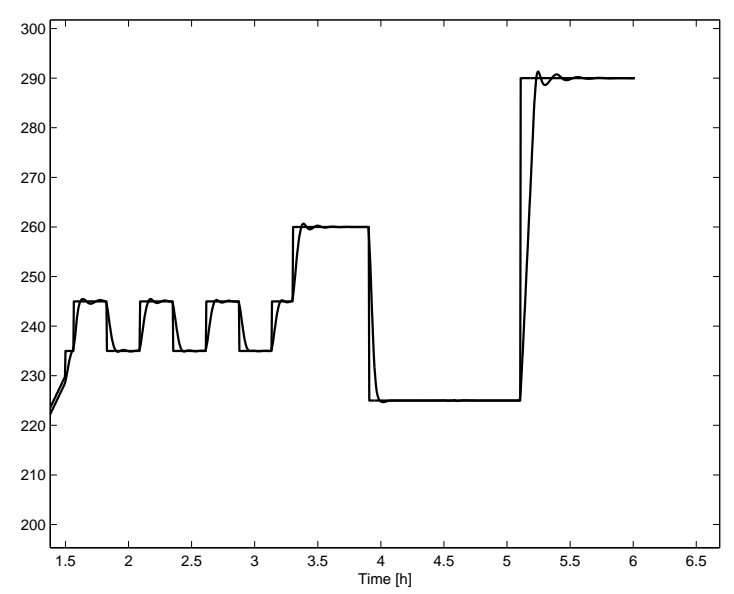

Fig. 12. Example 3 - Detail of temperature response.

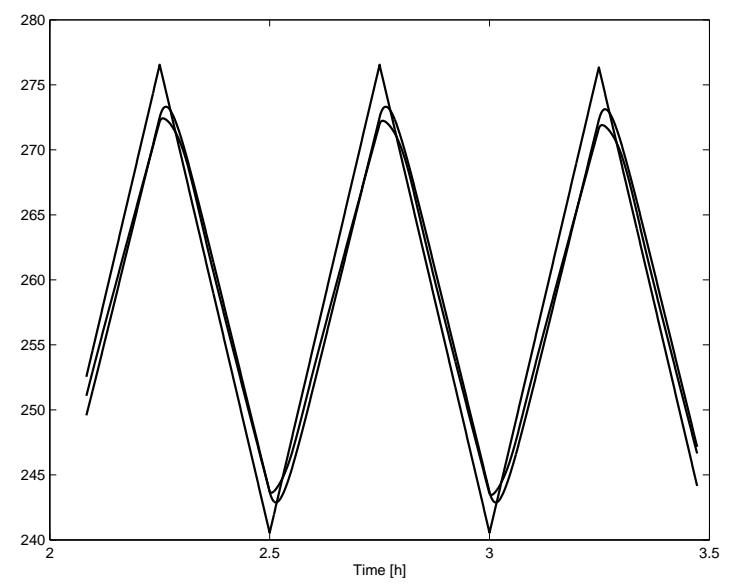

Fig. 13. Linearized system response to a square wave.

extra filter $\left(K_{p}=0.96, \tau=75 s\right)$, between $2 h$ and $3.4 h$, for a square wave $(u(t)=0.04 \operatorname{sign}[\sin (3.5 \times$ $\left.\left.10^{-3} t\right)\right]$ ), as well as the result of coupling (2) with (8. The improved approximation of the integrator with extra filter is clear. Fig. 14 shows two iterations for the computation of NLM, one with the pure integrator model (dashed) and the other with the integrator with an extra filter. The filter provides an improvement of $80 \%$. The results obtained in other experiments are consistent, thereby justifying the proposed model.

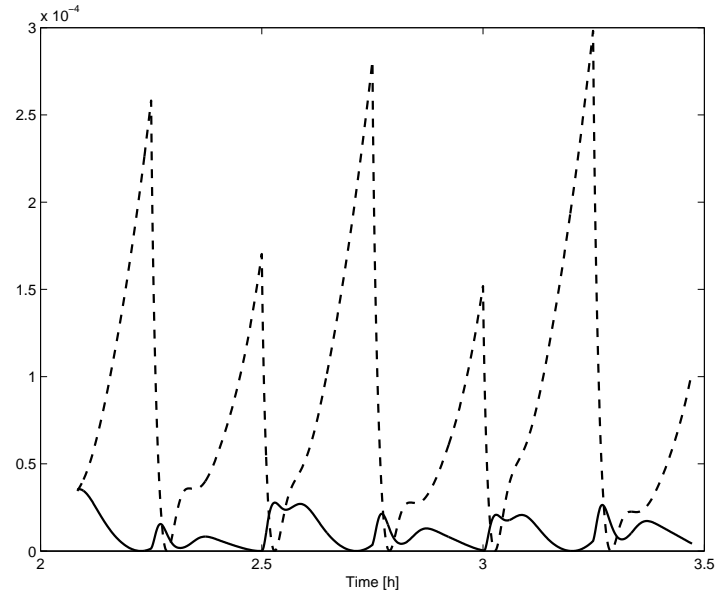

Fig. 14. Two iterations of NLM with the pure integrator (dashed) and the filtered integrator (solid).

\section{REFERENCES}

Barão M., J. M. Lemos and R. N. Silva (2002). Reduced complexity adaptative nonlinear control of a distributed collector solar field, J. Proc. Control, 12:131-141.

Camacho, E. F., M. Berenguel and C. Bordóns (1994). Adaptive Generalized Predictive Control of a Distributed Collector Field. IEEE Trans. Control Syst. Tech., 2(4):462-467.

Camacho, E. F., M. Berenguel and F. Rubio (1997). Advanced Control of Solar Plants, SpringerVerlag.

Coito, F., J. M. Lemos, R. N. Silva and E. Mosca (1997). Adaptive control of a solar energy plant: Exploiting accessible disturbances. Int. J. Adaptive Control and Signal Proc., 11(4):327-342.

Isidori, A. (1995). Nonlinear Control Systems, Springer-Verlag, 3rd Ed..

Mosca, E. (1995). Optimal, Predictive and Adaptive Control, Prentice Hall.

Schweickhardt, T. and F. Allgöwer (2003). Quantitative nonlinearity assessment - An introduction to nonlinearity measures, in M. Georgiadis and P. Seferlis, eds., The Integration of Design and Control, Computer-aided chemical engineering. Elsevier Science.

Schweickhardt, T., F. Allgöwer, F. and F. J. Doyle III (2003). Nonlinearity Quantification for the Optimal State Feedback Controller, Proc. EEC 03, European Control Conference.

Silva, R. N., J. M. Lemos and L. M. Rato (2003). Variable Sampling Adaptive Control of a Distributed Collector Solar Field, IEEE Trans. Control Syst. Tech., 11(5):765-772. 